\title{
A brave new world: novel non-training posts to support career breaks, reduce rota gaps and reduce locum spend
}

\author{
Author: Thomas Livingston
}

\section{Background}

Nottingham University Hospitals NHS Trust is suffering with significant junior doctor rota gaps; a result of the imposition of the new junior doctor contract, poor recruitment to core medical training and an increasing trend towards trainees taking a career break post-completion of the foundation programme $58 \%$ in 2017). These gaps are impacting on training opportunities and morale of trainees, and operationally are leaving wards shortstaffed resulting in an increased reliance on locum doctors, particularly out of hours. We identified that there were 1,286 rota gaps in the foundation doctor and core trainee rota from February 2017 to February 2018, which equated to around 10,000 hours. Only $52 \%$ of these gaps were filled with locum doctors at a cost of nearly $£ 900,000$ (Fig 1), with $72 \%$ of these shifts being out of hours. This is likely to be exacerbated in 2018 by the removal of six core medical training posts by Health Education East Midlands.

Aims

> To improve recruitment of trainees post-completion of the UK foundation programme to non-training posts.

$>$ To reduce rota gaps by $50 \%$.

> To reduce locum spend by $50 \%$.

\section{Methods}

We devised two novel non-training programmes aimed at trainees post-completion of the UK foundation programme, who are likely to take a career break and who do not currently apply for our established trust grade posts. These will incorporate:

'Foundation year 3' posts offering:

> eight months service provision paid over an annualised contract (creating an effective 4 month paid sabbatical) and equating to eight whole time equivalent posts

> protected half-day training time for a special interest and study budget

> flexible hours and option to contribute $60-100 \%$ to the on-call rota.

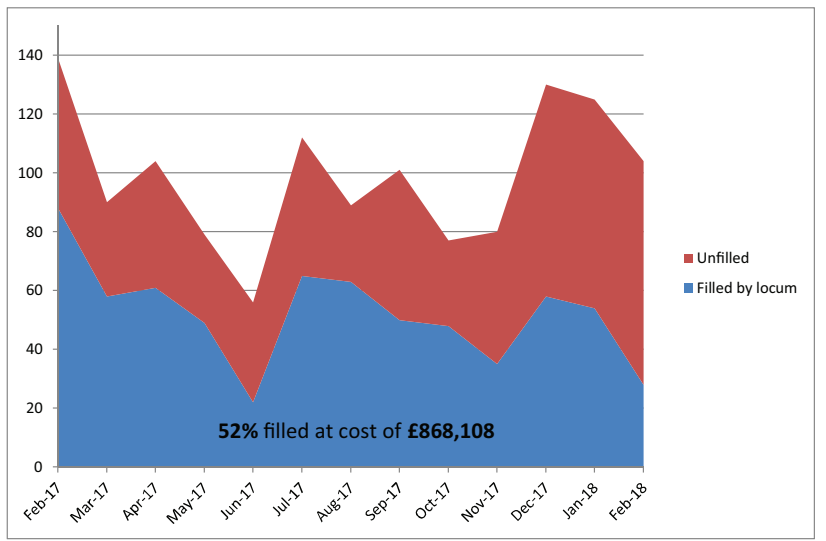

Fig 1. F1 to CT2 rota gaps from February 2017 to February 2018

\section{Hospital at night posts offering:}

> one week per month protected non-clinical time for service development

$>$ flexible out-of-hours working aimed at covering current rota gaps

$>$ study budget and annual leave.

Results

We will measure the success of this programme by assessing:

$>$ total number of applicants and competition ratios

$>$ percentage monthly decrease in rota gaps

$>$ percentage monthly decrease in locum spend

> subjective feedback survey.

Results will be updated as it becomes available. Initial data will be available for June 2018 (competition ratios). Rota gap and locum spend data will be collected from August 2018.

\section{Conflict of interest statement}

None. 